\title{
SHORT REPORT \\ The first study on opportunistic intestinal microsporidiosis in IBD patients receiving immunosuppressive medications in Iran
}

\author{
Z. HASANI ${ }^{1}$, H. ASADZADEH AGHDAEI ${ }^{2}$, H. BALAII $^{2}$, M. AZIMIRAD ${ }^{1}$, \\ E. S. MIRSAMADI ${ }^{3}$, H. MIRJALALI ${ }^{1}$ AND M.R. ZALI ${ }^{4}$ \\ ${ }^{1}$ Foodborne and Waterborne Diseases Research Center, Research Institute for Gastroenterology and Liver \\ Diseases, Shahid Beheshti University of Medical Sciences, Tehran, Iran \\ ${ }^{2}$ Basic and Molecular Epidemiology of Gastrointestinal Disorders Research Center, Research Institute for \\ Gastroenterology and Liver Diseases, Shahid Beheshti University of Medical Sciences, Tehran, Iran \\ ${ }^{3}$ Department of Microbiology, Faculty of Medicine, Shahid Beheshti University of Medical Sciences, Tehran, Iran \\ ${ }^{4}$ Gastroenterology and Liver Diseases Research Center, Research Institute for Gastroenterology and Liver \\ Diseases, Shahid Beheshti University of Medical Sciences, Tehran, Iran
}

Received 21 August 2016; Final revision 11 March 2017; Accepted 12 April 2017;

first published online 15 May 2017

\section{SUMMARY}

Microsporida are known as opportunistic unicellular organisms and have recently been reclassified as fungi that have been frequently reported from patients with congenital and acquired immunity failure disorders, worldwide. However, use of immunosuppressive medications in inflammatory bowel disease (IBD) patients significantly decreases overall immunity, and increases their susceptibility to opportunistic infections. Totally, 71 stool samples were collected from IBD patients consisted of 69 ulcerative colitis (UC) patients and two Crohn's disease (CD) patients. All patients had taken immunosuppressive and/or immunomodulator drugs for at least 3 weeks. DNA was extracted from all stool samples and Nested PCR was performed using genusspecific primers based on small subunit ribosomal RNA (SSU rRNA) gene. Fisher's Exact Test was applied to evaluate statistical association between microsporidia infection and sex, age and types of IBD. Mean of age \pm S.D., women and men percentage of the attended patients were $36 \cdot 17 \pm 11 \cdot 93,60 \cdot 6 \%$, and $39 \cdot 4 \%$, respectively. A 440-bp fragment of SSU rRNA gene attributed to Enterocytozoon bieneusi was amplified from $12 \cdot 7 \%$ of IBD patients. No Encephalitozoon DNA was detected in the samples. No microsporidia-positive sample was found in CD patients. Fisher's Exact Test showed that there was no statistically significant correlation between intestinal microsporidiosis and age, sex, and IBD types with $P$ values: $0 \cdot 389,1 \cdot 00$, and $1 \cdot 00$, respectively. This study has shown IBD patients undergoing immunosuppressive/immunomodulators medications, which may be susceptible to intestinal microsporida infection. E. bieneusi is the commonest intestinal microsporidan reported from IBD patients.

Key words: Enterocytozoon bieneusi, IBD patients, immunosuppressive medication, Iran, opportunistic infection.

\footnotetext{
* Author for correspondence: Dr H. Mirjalali, Foodborne and Waterborne Diseases Research Center, Research Institute for Gastroenterology and Liver Diseases, Shahid Beheshti University of Medical Sciences, Tehran, Iran. (Email: hamed_mirjalali@hotmail.com)
}

Microsporida are known as opportunistic unicellular organisms and have recently been reclassified as fungi [1]. This microorganism has frequently been reported from both immunocompromised and 
immunocompetent individuals, as well as a broad range of animals all over the world [2, 3]. Microsporidia infection in immunocompromised patients, particularly HIV/AIDS patients, is one of the most important concerns of healthcare systems. Reports of microsporida infection have risen worldwide due to the increase in congenital/acquired immunity system disorders [4]. Enterocytozoon bieneusi is one of the most prevalent human-infecting microsporidan species reported from HIV/AIDS patients [5], patients undergoing chemotherapy [6], and individuals with immunity disorders.

Inflammatory bowel disease (IBD) is a complex gastrointestinal disorder and the etiological agent is not clearly known. Crohn's disease (CD) and ulcerative colitis (UC) are the two most important chronic IBD, frequently reported worldwide [7, 8]. Several genetic, environmental, and immunological factors have been suggested as triggers of immunological disorders and subsequent IBD. Nowadays, immunosuppressive/immunomodulatory agents such as corticosteroids and anti-inflammatory drugs are used to manage conditions of IBD patients [7]. However, the increasing use of immunosuppressive drugs in IBD patients, significantly decreases overall immunity and increases susceptibility to opportunistic infections. However, subsequent events of immunosuppressive therapy in IBD patients convinced the ECCF (European Crohn's and Colitis Foundation) to state that IBD patients on corticosteroids, biological agents, and immunomodulators are normally more susceptible to opportunistic pathogens [9].

Several studies have presented different opportunistic microbial pathogens from IBD patients [10]. Since intestinal microsporidiosis is considered as an opportunistic infection, frequently reported from immunocompromised patients, IBD patients undergoing immunosuppressive drugs could be more susceptible to this infection.

However, the current study is the first survey to investigate intestinal microsporidiosis in IBD patients undergoing immunosuppressive therapy.

Totally, 71 stool samples were collected from IBDs patients consisted of $69 \mathrm{UC}$ patients and two CD patients who had been referred to IBD clinic of Research Institute for Gastrointestinal and Liver Diseases in Shahid Beheshti University of Medical Sciences, Tehran during May 2015 to April 2016. A trained interviewer filled up a questionary consisted of age, sex, and type of IBD. All patients had taken immunosuppressive and/or immunomodulator drugs for at least 3 weeks. Stool samples were immediately transferred to the Parasitology Laboratory of Foodborne and Waterborne Diseases Research Center, Research Institute for Gastroenterology and Liver Diseases in Shahid Beheshti University of Medical Sciences and further investigations were carried out on the samples.

DNA extraction was performed for all stool samples using QIAamp DNA Stool mini Kit according to the manufacturer's instruction. Nested PCR was performed using genus-specific primers based on small subunit ribosomal RNA (SSU rRNA) gene according to the literature mentioned elsewhere [5]. Briefly, the first pair primers, PMicF (5' - GGTTGATTCTGCCTGACG $\left.3^{\prime}\right)$ and PMicR (5' - CTTGCGAGC(G/A)TACTATCC - $3^{\prime}$ ), amplified $779 \mathrm{bp}$ of SSU rRNA gene of Encephalitozoon spp. and Enterocytozoon bieneusi. The second PCR employed primers EnbF (5' GGTAATTTGGTCTCTGTGTG - 3') and EnbR $\left(5^{\prime}\right.$ - CTACACTCCCTATCCGTTC $\left.-3^{\prime}\right)$ to amplify $440 \mathrm{bp}$ as well as EncepF (5' - AGTACGATGATTT GGTTG - 3') and EncepR (5' - ACAACACTATAT AGTCCCGTC $-3^{\prime}$ ) to amplify $629 \mathrm{bp}$ fragments for E. bieneusi and Encephalitozoon spp., respectively.

First, PCR reaction was performed in final volume $25 \mu \mathrm{l}$ containing $12 \cdot 5 \mu \mathrm{l}$ of $2 \times$ Ampliqon PCR Master Mix (Denmark) $10 \rho \mathrm{M}$ of each primers. Amplifications were carried out in Eppendorf thermocycler (Hamburg, Germany). The conditions for the first PCR reaction were: $95^{\circ} \mathrm{C}$ for $5 \mathrm{~min}$ followed by 35 cycles of $94{ }^{\circ} \mathrm{C}$ for $40 \mathrm{~s}, 55^{\circ} \mathrm{C}$ for $45 \mathrm{~s}, 72^{\circ} \mathrm{C}$ for $45 \mathrm{~s}$, and final extension of $72^{\circ} \mathrm{C}$ for $4 \mathrm{~min}$. The second PCR conditions were: $95^{\circ} \mathrm{C}$ for $5 \mathrm{~min}$ followed by 25 cycles of $94^{\circ} \mathrm{C}$ for $35 \mathrm{~s}, 57^{\circ} \mathrm{C}$ for $35 \mathrm{~s}, 72{ }^{\circ} \mathrm{C}$ for $40 \mathrm{~s}$, and $72{ }^{\circ} \mathrm{C}$ for $3 \mathrm{~min}$ as a final extension. The $10 \mu \mathrm{l}$ of PCR products were electrophoresed on $1.5 \%$ of agarose gel and were visualized after ethidium bromide staining. A positive (sequenced isolates with accession no. KJ414444) and negative (Sterile Distillated Water) control were run together with all samples.

Fisher's Exact Test was applied to evaluate statistical association between microsporidia infection and sex, age, and type of IBD using IBM SPSS Statistics for Windows, v22 (Chicago, IL, USA). A $P$-value $<0.05$ was considered statistically significant.

Totally, 71 patients including $69(97 \cdot 18 \%)$ and 2 $(2 \cdot 82 \%)$ had UC and CD, respectively. IBD and type of IBD were proven in all patients using by colonoscopy and pathology evaluations. Mean of age \pm S.D., women and men percentage of the attended patients were $36 \cdot 17 \pm 11 \cdot 93,60 \cdot 6 \%$ and $39 \cdot 4 \%$, 
Table 1. Demographic and summarized data of microsporidia infected patients among IBD subjects

\begin{tabular}{|c|c|c|}
\hline & & $P$-value \\
\hline Microsporidia-positive patients & $9 / 71$ & 0 \\
\hline \multicolumn{3}{|l|}{ Microsporidia species } \\
\hline Enterocytozoon bieneusi & 9 & \\
\hline Encephalitozoon spp. & 0 & 0 \\
\hline \multicolumn{3}{|l|}{ Age } \\
\hline Mean of age \pm s.D. of enrolled patients & $36 \cdot 17 \pm 11 \cdot 93$ & \\
\hline Mean of age \pm s.D. of infected patients & $36 \cdot 44 \pm 8 \cdot 56$ & $0 \cdot 389$ \\
\hline \multicolumn{3}{|l|}{ Gender } \\
\hline Male & $3 / 28$ & \\
\hline Female & $6 / 43$ & $1 \cdot 00$ \\
\hline \multicolumn{3}{|l|}{ IBDs type } \\
\hline Ulcerative colitis & $69 / 71$ & \\
\hline Crohn's disease & $2 / 71$ & $1 \cdot 00$ \\
\hline \multicolumn{3}{|c|}{ Immunosuppressive/immunomodulator medication in infected patients } \\
\hline Methyl-prednisolone & $9 / 9$ & \\
\hline Hydrocortisone & $0 / 9$ & \\
\hline Mesalamine & $0 / 9$ & \\
\hline Infliximab & $0 / 9$ & $-*$ \\
\hline
\end{tabular}

* $P$-value was not applicable.

respectively. All demographic data are summarized in Table 1. All enrolled patients had received one or several following drugs: Methyl-prednisolone or hydrocortisone, infliximab and mesalamine at least for 3 weeks. All patients had watery diarrhea or semi-form stool at the time of sampling.

PCR reactions were carried out on all DNA extracted samples and nine (12.7\%) samples were identified as positive. All nine positive samples showed $440 \mathrm{bp}$ fragment of SSU rRNA gene attributed to $E$. bieneusi, while no DNA amplification was found by specific primers for Encephalitozoon spp. Furthermore, all $E$. bieneusi positive-samples were seen in UC patients $(13 \cdot 4 \%)$, while no positive sample was found in CD patients. Statistical analysis was performed and Fisher's Exact Test showed that there was no statistically significant correlation between intestinal microsporidiosis and age, sex, and IBD types with $P$-values: $0 \cdot 389,1 \cdot 00$, and $1 \cdot 00$, respectively.

Microsporida infection is one of the most important concerns and complications of physicians treating immunocompromised patients. Recently, gastrointestinal microsporidiosis was described as an emerging infectious disease reported from immunocompromised patients [5].

Nowadays, increase in the number of immune system disorders has led to opportunistic microorganisms being considered as one of the main secondary problems in immunocompromised patients. Several opportunistic microorganisms have been reported from IBD patients on corticosteroids, immunomodulators, and biological agents. Although the co-existence of some viruses, bacteria, and fungi were represented, there are scarce data available on opportunistic parasites in IBD patients [10].

Studies have reported the opportunistic infection of intestinal microsporidia from almost all countries [2]. However, gastrointestinal microsporidiosis not only decreases the life-quality of IBD patients, but also increases the intricacy of the therapy process. The current study is the first to report gastrointestinal microsporidiosis in IBD patients undergoing immunosuppressive therapy. Recently, Andreu-Ballester et al. suggested an etiological role for microsporidia in CD patients before immunosuppression and stated that due to deficiencies of $\delta \gamma$ T lymphocytes and IL-7, CD patients are likely to be more susceptible to colonization of intestinal microsporida. In contrast with our study, all enrolled patients in mentioned research had not been treated for $\mathrm{CD}$ and had not undergone immunosuppression therapy [11].

The prevalence rate of the microsporidia infection in the current study was $12.7 \%$. In Iran, there are studies that described intestinal microsporidia from HIV/ AIDS patients [5, 12], cancer patients under chemotherapy and transplant recipients [13]. A previous study from Iran by Mirjalali et al. showed high prevalence $(30 \cdot 86 \%)$ of E. bieneusi among HIV/ AIDS patients, while some other studies reported 
lower rates of the infection [5]. Agholi et al. found $E$. bieneusi from $9 \cdot 55 \%$ of HIV/AIDS patients and $6.81 \%$ of transplant recipient children in the south of Iran $[12,14]$. In another study, Tabatabaie et al. reported prevalence rates of $5 \cdot 3 \%$ and $4 \%$ for E. bieneusi and Encephalitozoon sp., respectively, in healthy subjects and $0.7 \%$ and $5.7 \%$ for E. bieneusi and Encephalitozoon sp., respectively, in various samples of immunocompromised patients [15]. In our study, only E. bieneusi was found in $12.7 \%$ of IBD patients who underwent immunosuppressive/immunomodulator medications. The prevalence rate of our study is similar and in agreement with other studies showing microsporidiosis in immunocompromised patients in Iran.

In the current study, only E. bieneusi was detected in all microsporidia-positive patients and no DNA of Encephalitozoon was amplified. Almost all previous studies in Iran showed that E. bieneusi had a higher prevalence rate $[3,5,12,14]$. On the other hand, the interesting point is that metronidazole consumption with or without prescription is common in IBD patients to prevent diarrhea. Although, He et al. showed that metronidazole could be effective against Encephalitozoon spp. [16], but E. bieneusi is perceived as resistant to benzimidazoles such as metronidazole [17]. Therefore, the low prevalence of Encephalitozoon spp., compared with E. bieneusi in IBD patients, as our results showed, could be predictable.

In this study, no positive sample was detected in $\mathrm{CD}$ patients. This finding is more likely related to the number of $\mathrm{CD}$ patients. In Iran, the prevalence rate of $\mathrm{UC}$ is more than the $\mathrm{CD}$ cases [18] and unfortunately, at the time of sampling the number of $\mathrm{CD}$ cases was very low. However, intestinal microsporidia, particularly E. bieneusi, should be considered as one of the most important opportunistic microorganisms, capable of increasing the complexity of managing IBD patients.

IBD patients undergoing immunosuppressive, corticosteroid, and immunomodulator agents could be susceptible to intestinal microsporida infection. In addition, E. bieneusi is the commonest microsporidian species reported from this group of IBD patients.

\section{ACKNOWLEDGEMENTS}

The authors would like to appreciate all colleagues of Foodborne and Waterborne Diseases Research Center for their laboratory cooperation.

\section{DECLARATION OF INTEREST}

None.

\section{REFERENCES}

1. Capella-Gutierrez S, Marcet-Houben M, Gabaldon T. Phylogenomics supports microsporidia as the earliest diverging clade of sequenced fungi. BMC Biology 2012; 10: 47.

2. Field AS, Milner DA. Jr. Intestinal microsporidiosis. Clinics in Laboratory Medicine 2015; 35(2): 445-459.

3. Askari Z, et al. Molecular detection and identification of zoonotic microsporidia spore in fecal samples of some animals with close-contact to human. Iranian Journal of Parasitology 2015; 10(3): 381-388.

4. Abdelmalek R, et al. [Microsporidia and cryptosporidia coinfection in an HIV-infected newborn]. Archives de Pediatrie: organe officiel de la Societe francaise de pediatrie 2011; 18(5): 562-564.

5. Mirjalali H, et al. Emerging intestinal microsporidia infection in HIV(+)/AIDS Patients in Iran: Microscopic and Molecular Detection. Iranian Journal of Parasitology 2014; 9(2): 149-154.

6. Lono AR, Kumar S, Chye TT. Incidence of microsporidia in cancer patients. Journal of Gastrointestinal Cancer 2008; 39(1-4): 124-129.

7. Ko JK, Auyeung KK. Inflammatory bowel disease: etiology, pathogenesis and current therapy. Current Pharmaceutical Design 2014; 20(7): 1082-1096.

8. Corridoni D, Arseneau KO, Cominelli F. Inflammatory bowel disease. Immunology Letters 2014; 161(2): 231-235.

9. Rahier JF, et al. Second European evidence-based consensus on the prevention, diagnosis and management of opportunistic infections in inflammatory bowel disease. Journal of Crohn's \& Colitis 2014; 8(6): 443-468.

10. Dave M, et al. Opportunistic infections due to inflammatory bowel disease therapy. Inflammatory Bowel Diseases 2014; 20(1): 196-212.

11. Andreu-Ballester JC, et al. Microsporidia and its relation to Crohn's disease. A retrospective study. PLoS ONE 2013; 8(4): e62107.

12. Agholi M, Hatam GR, Motazedian MH. HIV/ AIDS-associated opportunistic protozoal diarrhea. AIDS Research and Human Retroviruses 2013; 29(1): 35-41.

13. Mirjalali H, et al. Genotyping and molecular analysis of Enterocytozoon bieneusi isolated from immunocompromised patients in Iran. Infection, Genetics and Evolution: Journal of Molecular Epidemiology and Evolutionary Genetics in Infectious Diseases 2015; 36: 244-249.

14. Agholi M, Hatam GR, Motazedian MH. Microsporidia and coccidia as causes of persistence diarrhea among liver transplant children: incidence rate and species/genotypes. The Pediatric Infectious Disease Journal 2013; 32(2): 185-187.

15. Tabatabaie F, et al. Molecular detection of microsporidiosis in various samples of Iranian immunocompromised patients. Journal of Parasitic Diseases: Official 
Organ of the Indian Society for Parasitology 2015; 39(4): 634-638.

16. He Q, et al. Effects of nifedipine, metronidazole, and nitric oxide donors on spore germination and cell culture infection of the microsporidia Encephalitozoon hellem and Encephalitozoon intestinalis. Antimicrobial Agents and Chemotherapy 1996; 40(1): 179-185.
17. Canning EU, Hollister WS. Enterocytozoon bieneusi (Microspora): prevalence and pathogenicity in AIDS patients. Transactions of the Royal Society of Tropical Medicine and Hygiene 1990; 84(2): 181-186.

18. Taghavi SA, et al. Epidemiology of inflammatory bowel diseases (IBD) in Iran: a review of 740 patients in Fars Province, Southern Iran. Annals of Colorectal Research 2013; 1(1): 17-22. 